\title{
Tracheo-innominate Artery Fistula in a Complicated Penetrating Neck Trauma: A Successfully Managed Rare Complication of Low Tracheotomy
}

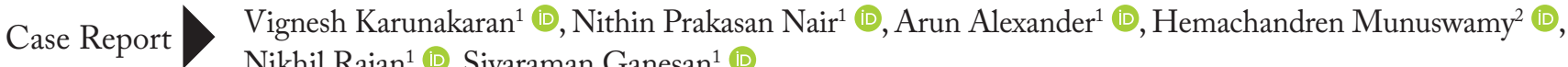 \\ Nikhil Rajan ${ }^{1}$ (D), Sivaraman Ganesan ${ }^{1}$ (D) \\ ${ }^{1}$ Department of Otorhinolaryngology, Jawaharlal Institute of Post Graduation Education and Research (JIPMER), Pondicherry, India \\ ${ }^{2}$ Department of Cardio Thoracic Vascular Surgery, Jawaharlal Institute of Post Graduation Education and Research (JIPMER), \\ Pondicherry, India
}

Abstract

\begin{abstract}
ORCID iDs of the authors:
V.K. 0000-0002-0850-441X

N.P.N. 0000-0001-7342-9682;

A.A. $0000-0003-1026-4678$;

H.M. 0000-0003-2597-084X;

N.R. $0000-0002-8982-473 X$

S.G. 0000-0002-7065-0258
\end{abstract}

Cite this article as: Karunakaran V, Nair NP, Alexander A, Munuswamy H, Rajan N, Ganesan S. Tracheo-innominate Artery Fistula in a Complicated Penetrating Neck Trauma: A Successfully Managed Rare Complication of Low Tracheotomy. Turk Arch Otorhinolaryngol 2020; 58(2): 127-9.

Corresponding Author:

Nithin Prakasan Nair, nithinprakasannair.2008@ gmail.com

Received Date: 15.12 .2019

Accepted Date: 08.03 .2020

Content of this journal is licensed under a Creative Commons Attribution 4.0 International License. Available online at www.turkarchotolaryngol.net
Tracheo-innominate artery fistula is a rare complication of tracheotomy with very high mortality rate. Only a few patients survive this complication as reported in the literature. Here we report the case of a 54-year-old gentleman who presented to the emergency department with a history of penetrating neck trauma following a road traffic accident. Neck exploration and tracheotomy were done to secure the airway. After two weeks, the patient had an episode of massive stomal bleed for which he was taken to the operating room and re-explored. A tracheo-in- nominate artery fistula was detected, and right side aorto-carotid and right side aorto-subclavian anastomoses were done using reversed saphenous vein graft with interruption of flow. Following a successful surgery, the patient was decannulated later, and now lives a healthy normal life. Early diagnosis and immediate intervention are the key in managing this complication. Bedside management also plays a vital role.

Keywords: Tracheotomy, hemorrhage, innominate artery, oesophageal perforation, complication

\section{Introduction}

Tracheotomy is a life-saving procedure. Tracheo-innominate artery fistula is an infrequent, delayed complication of tracheotomy (1). It is associated with high mortality rates and catastrophic for the surgeon. Any complaints of bleeding from the site after 14 days of tracheotomy should always arouse suspicion of tracheo-innominate artery fistula and must be evaluated meticulously (2).

Here, we report a case of tracheo-innominate artery fistula, that was successfully managed.

\section{Case Presentation}

A 54-year-old man presented to the emergency room with penetrating neck trauma and stable vitals. There was no history suggestive of head injury. Further evaluation revealed subcutaneous emphysema around the neck and the chest. There was a $4 \times 2 \mathrm{~cm}$ laceration in the mid-neck, exposing the trachea. An X-ray revealed air shadow in the retropharyngeal space and the pneumomediastinum. Informed consent was obtained, and emergen- cy tracheotomy with neck exploration was done. There was no great vessel or oesophageal injury. A rent was noted on the anterior tracheal wall and converted into tracheostoma. Nasogastric tube feeding was begun postoperatively.

Non-contrast computed tomography (CT) of the neck for progressing postoperative neck emphysema revealed disrupted tracheal cartilage at the level of the tracheotomy. The tube appeared to be hinging on the posterior tracheal wall. Neck re-exploration was done on the $6^{\text {th }}$ postoperative day. The tracheotomy tube was indeed found to be hinging on the posterior tracheal wall. There was no obvious rent on the posterior tracheal wall. A longer tracheotomy tube was inserted through the same stoma. Subcutaneous emphysema regressed after this intervention, however, the patient did not tolerate oral intake and continued to receive nasogastric tube feeding.

On the $19^{\text {th }}$ postoperative day, the patient had mild blood-tinged secretions from the tracheotomy tube, which a few hours later developed into tor- 
rential bleeding. Immediately the tracheotomy tube cuff was hyperinflated with $40 \mathrm{~mL}$ of air. Utley's maneuver was applied and the bleeding was temporarily controlled. Resuscitation measures were started with crystalloids and blood products. The patient was taken for emergency exploration.

Median sternotomy revealed a tracheo-innominate artery fistula. The left saphenous vein was harvested, reversed and used to anastomose the right common carotid distally and the aorta proximally. Proximal to the anastomosis, the carotid artery was ligated (Figure 1). Another reversed saphenous vein anastomosis was done between the right subclavian and the aorta. The part proximal to the subclavian anastomosis was also ligated (Figure 2). The origin of the innominate artery was looped and ligated. The rent noted in the posterior trachea was sutured. The deficient anterior tracheal wall was closed with a thymic pedicle flap. The oesophageal perforation that was noted intraoperatively was repaired. A new stoma was made and a tracheotomy tube was inserted. Feeding jejunostomy was done. The patient received broad-spectrum antibiotics with anticoagulation. The patient was discharged on the 35th day of admission.

The patient was regularly followed up. CT angiography was performed in his sixth-month evaluation, and both anastomotic segments appeared to be patent (Figure 3). Barium swallow evaluation showed no evidence of tracheo-oesophageal fistula. The patient was started on oral feeding and weaned off the feeding tubes. Decannulation was done after the vocal cords and the airway were assessed and found normal. After two years of follow-up, the patient is alive and healthy.

\section{Discussion}

Hemorrhage is a common complication of tracheotomy occurring in almost $5 \%$ of all tracheostomies. Tracheo-innominate artery fistula is a life-threatening delayed complication of tracheotomy, generally noted following the third week of the procedure (1). It is usually associated with high mortality rates. In 1924 , Schlaepfer (3) reported a mortality rate of $73 \%$ in a case series of 115 patients.

There are many mechanisms proposed for this complication. It could be due to a low tracheotomy, in which the tube would be just overlying the innominate artery. It can be because of the tube end that hinges on the anterior tracheal wall. Another proposed mechanism is the pressure necrosis due to a hyperinflated cuff. In our case, it may be due to low tracheotomy or long tube

\section{Main Points}

- Tracheo innominate artery fistula is a rare delayed complication of tracheotomy with high mortality rates.

- Bleeding from tracheotomy side after 14 days should be considered probable fistula and should be evaluated with CT angiography.

- Immediate bedside management measures like hyperinflating the cuff, applying pressure, clearing the airway and replacement of fluids with blood will buy more time before taking the patient to operating room. that hinged on the anterior tracheal wall. Low tracheotomy and peristomal granulations are considered as risk factors for tracheo-innominate fistula (4).

Herald bleeding, any bleeding via the tracheotomy tube in the form of blood-tinged secretion, or frank bleeding can be an alarming sign. Pulsating tracheotomy cannula and hemoptysis should be considered as herald events (2). Such alarms should prompt further evaluation immediately. Early diagnosis can reduce mortality. A CT angiography can help identify any fistula (5).

Early bedside management can be life-saving for the patient. Hyperinflation of the cuff may help to seal off the fistula site temporarily (6). Suctioning and clearing the airway is also equally important. Fluid resuscitation with crystalloids and blood products is critical and must be started urgently (7). We believe we saved this patient mainly because of early bedside management measures.

Digital pressure via pretracheal fascia against the sternum, which is known as the Utley's maneuver, can be life-saving by stopping bleeding at the bedside (8). Definitive management can be by either open or endovascular procedures. Open procedures can be done following a median sternotomy by ligation/resection of the tracheo-innominate artery or by maintaining the flow via interposition. In our case, we have ligated the tracheo-innominate artery and bypassed the circulation. The possible complication of

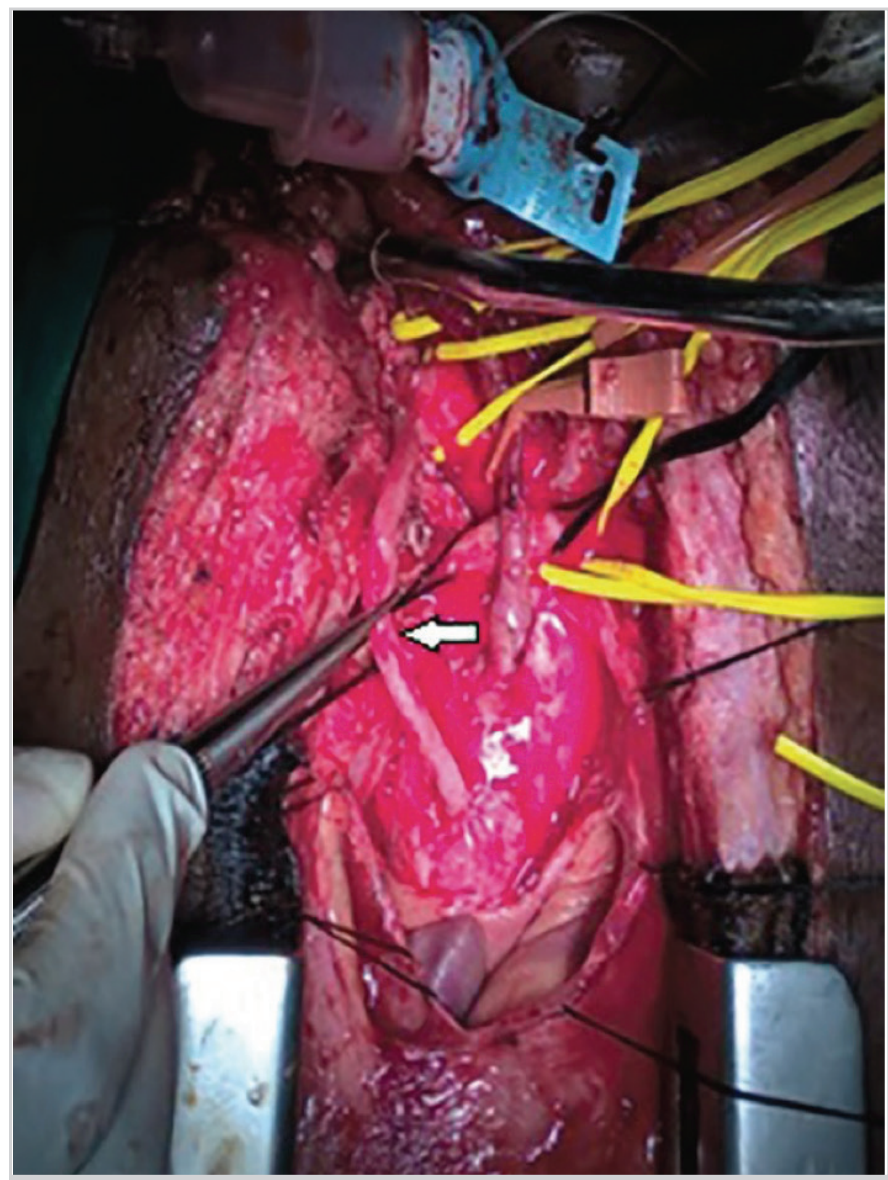

Figure 1. Anastomosis of right carotid aorta with reversed saphenous vein (white arrow) 


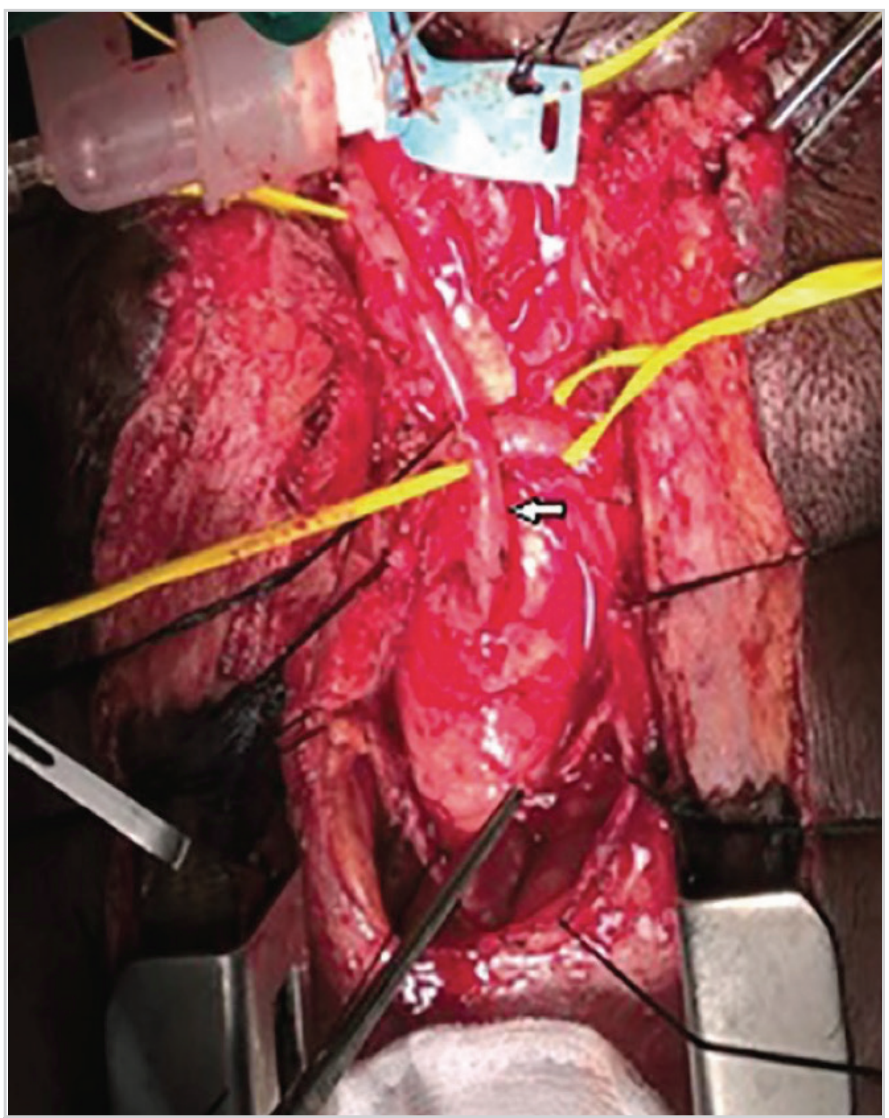

Figure 2. Anastomosis of right subclavian aorta with reversed saphenous vein (white arrow)

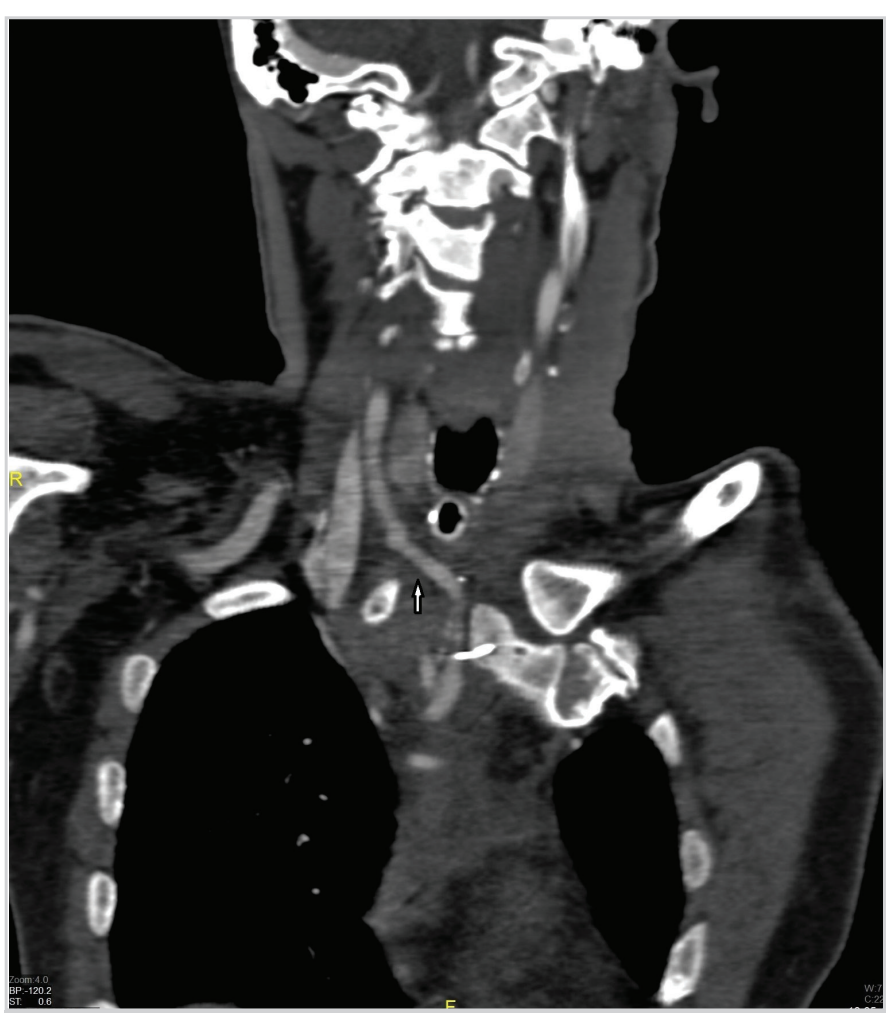

Figure 3. Computed tomography angiography done at sixth-month follow-up showing patent anastomosis (white arrow)

bypassing the circulation is ischemic stroke. Endovascular stenting has also been tried in poor surgical candidates with varying success rates (9).
Gelman et al. (10) reviewed 71 survivors of tracheo-innominate artery fistula from 1962 to 1994 . Of the 71 initial survivors, only 40 survived "long-term" (>2 months). Only $15.8 \%$ of the patients with maintenance of flow had "long-term" survival as compared to $71.2 \%$ in patients with interruption of flow. Early diagnosis and definitive management are the keys to saving a patient with tracheo-innominate artery fistula.

\section{Conclusion}

Even though tracheo-innominate artery fistulas have a high mortality rate, early diagnosis can prevent this outcome. In any minor suspicion of a tracheo-innominate artery fistula, CT angiography should be done. Presence of mind at the time of catastrophic bleeding may also save the patient. Utley's maneuver, hyperinflating the cuff and fluid resuscitation are some bedside measures, which help us to buy time. Ultimately surgical management is the key to saving the patient.

Informed Consent: Informed consent was obtained from the patient.

Peer-review: Externally peer-reviewed.

Author Contributions: Concept - V.K.; Supervision - A.A., H.M.; Data Collection and/or Processing - V.K., N.P.N.; Literature Search - A.A., S.G.; Writing - N.P.N., N.R.; Critical Reviews - N.R., S.G.

Conflict of Interest: The authors have no conflicts of interest to declare.

Financial Disclosure: The authors declared that this study has received no financial support.

\section{References}

1. Fernandez-Bussy S, Mahajan B, Folch E, Caviedes I, Guerrero J, Majid A. Tracheotomy tube placement: early and late complications. J Bronchology Interv Pulmonol 2015; 22: 357-64. [Crossref]

2. Shamji FM, Deslauriers J, Nelems B. Recognition and management of life-threatening tracheovascular fistulae and how to prevent them. Thorac Surg Clin 2018; 28: 403-13. [Crossref]

3. Schlaepfer K. Fatal hemorrhage following tracheotomy for laryngeal diphtheria. JAMA 1924; 82: 1581-3. [Crossref]

4. Wang XL, Xu ZG, Tang PZ, Yu Y.Tracheo-innominate artery fistula: diagnosis and surgical management. Head Neck 2013; 35: 1713-8. [Crossref]

5. Komatsu T, Sowa T, Fujinaga T, Handa N, Watanabe H. Tracheo-innominate artery fistula: two case reports and a clinical review. Ann Thorac Cardiovasc Surg 2013; 19: 60-2. [Crossref]

6. Jones JW, Reynolds M, Hewitt RL, Drapanas T. Tracheo-innominate artery erosion: successful surgical management of a devastating complication. Ann Surg 1976; 184: 194-204. [Crossref]

7. Bradley PJ. Bleeding around a tracheotomy wound: what to consider and what to do? J Laryngol Otol 2009; 123: 952-6. [Crossref]

8. Menen RS, Pak JJ, Dowell MA, Patel AR, Ashiku SK, Velotta JB. Treatment of tracheoinnominate fistula with ligation of the innominate artery: a case report. Perm J 2016;20: 15-16. [Crossref]

9. Ridley RW, Zwischenberger JB. Tracheoinnominate fistula: surgical management of an iatrogenic disaster. J Laryngol Otol 2006; 120: 676-80. [Crossref]

10. Gelman JJ, Aro M, Weiss SM. Tracheo-innominate artery fistula. J Am Coll Surg 1994; 179: 626-34. 